Preprint

\title{
NEW CONGRUENCES INVOLVING PRODUCTS OF TWO BINOMIAL COEFFICIENTS
}

\author{
GUO-SHUAI MAO AND ZHI-WEI SUN
}

Abstract. Let $p>3$ be a prime and let $a$ be a positive integer. We show that if $p \equiv 1(\bmod 4)$ or $a>1$ then

$$
\sum_{k=0}^{\left\lfloor\frac{3}{4} p^{a}\right\rfloor} \frac{\left(\begin{array}{c}
2 k \\
k
\end{array}\right)^{2}}{16^{k}} \equiv\left(\frac{-1}{p^{a}}\right)\left(\bmod p^{3}\right)
$$

with (-) the Jacobi symbol, which confirms a conjecture of Z.-W. Sun. We also establish the following new congruences:

$$
\begin{aligned}
& \sum_{k=0}^{(p-1) / 2} \frac{\left(\begin{array}{c}
2 k \\
k
\end{array}\right)\left(\begin{array}{c}
3 k \\
k
\end{array}\right)}{27^{k}} \equiv\left(\frac{p}{3}\right) \frac{2^{p}+1}{3}\left(\bmod p^{2}\right), \\
& \sum_{k=0}^{(p-1) / 2} \frac{\left(\begin{array}{c}
6 k \\
3 k
\end{array}\right)\left(\begin{array}{c}
3 k \\
k
\end{array}\right)}{(2 k+1) 432^{k}} \equiv\left(\frac{p}{3}\right) \frac{3^{p}+1}{4}\left(\bmod p^{2}\right), \\
& \sum_{k=0}^{(p-1) / 2} \frac{\left(\begin{array}{c}
4 k \\
2 k
\end{array}\right)\left(\begin{array}{c}
2 k \\
k
\end{array}\right)}{(2 k+1) 64^{k}} \equiv\left(\frac{-1}{p}\right) 2^{p-1}\left(\bmod p^{2}\right) \text {. }
\end{aligned}
$$

Note that in 2003 Rodriguez-Villeguez posed conjectures on

$$
\sum_{k=0}^{p-1} \frac{\left(\begin{array}{c}
2 k \\
k
\end{array}\right)}{16^{k}}, \sum_{k=0}^{p-1} \frac{\left(\begin{array}{c}
2 k \\
k
\end{array}\right)\left(\begin{array}{c}
3 k \\
k
\end{array}\right)}{27^{k}}, \sum_{k=1}^{p-1} \frac{\left(\begin{array}{c}
4 k \\
2 k
\end{array}\right)\left(\begin{array}{c}
2 k \\
k
\end{array}\right)}{64^{k}}, \sum_{k=1}^{p-1} \frac{\left(\begin{array}{c}
6 k \\
3 k
\end{array}\right)\left(\begin{array}{c}
3 k \\
k
\end{array}\right)}{432^{k}}
$$

modulo $p^{2}$ which were later proved.

Key words and phrases. Central binomial coefficients, congruences.

2010 Mathematics Subject Classification. Primary 11B65, 11B68; Secondary 05A10, $11 \mathrm{~A} 07$.

The second author is the corresponding author. This research was supported by the Natural Science Foundation of China (grant 11571162). 


\section{INTRODUCTION}

Let $p>3$ be a prime. In 2003, via his analysis of the p-adic analogues of Gaussian hypergeometric series and the Calabi- Yau manifolds, Rodriguez-Villegas [RV] conjectured the following congruences:

$$
\begin{gathered}
\sum_{k=0}^{p-1} \frac{\left(\begin{array}{c}
2 k \\
k
\end{array}\right)^{2}}{16^{k}} \equiv\left(\frac{-1}{p}\right)\left(\bmod p^{2}\right), \sum_{k=0}^{p-1} \frac{\left(\begin{array}{c}
2 k \\
k
\end{array}\right)\left(\begin{array}{c}
3 k \\
k
\end{array}\right)}{27^{k}} \equiv\left(\frac{p}{3}\right)\left(\bmod p^{2}\right), \\
\sum_{k=0}^{p-1} \frac{\left(\begin{array}{c}
4 k \\
2 k
\end{array}\right)\left(\begin{array}{c}
2 k \\
k
\end{array}\right)}{64^{k}} \equiv\left(\frac{-2}{p}\right)\left(\bmod p^{2}\right), \sum_{k=0}^{p-1} \frac{\left(\begin{array}{c}
6 k \\
3 k
\end{array}\right)\left(\begin{array}{c}
3 k \\
k
\end{array}\right)}{432^{k}} \equiv\left(\frac{-1}{p}\right)\left(\bmod p^{2}\right),
\end{gathered}
$$

where $(\dot{\bar{p}})$ denotes the Jacobi symbol. They were soon proved by E. Mortenson [M1, M2] via the Gross-Koblitz formula and the $p$-adic $\Gamma$ function. Note that

$$
\begin{gathered}
\left(\begin{array}{c}
-1 / 2 \\
k
\end{array}\right)^{2}=\frac{\left(\begin{array}{c}
2 k \\
k
\end{array}\right)^{2}}{16^{k}},\left(\begin{array}{c}
-1 / 3 \\
k
\end{array}\right)\left(\begin{array}{c}
-2 / 3 \\
k
\end{array}\right)=\frac{\left(\begin{array}{c}
2 k \\
k
\end{array}\right)\left(\begin{array}{c}
3 k \\
k
\end{array}\right)}{27^{k}}, \\
\left(\begin{array}{c}
-1 / 4 \\
k
\end{array}\right)\left(\begin{array}{c}
-3 / 4 \\
k
\end{array}\right)=\frac{\left(\begin{array}{c}
4 k \\
2 k
\end{array}\right)\left(\begin{array}{c}
2 k \\
k
\end{array}\right)}{64^{k}}, \quad\left(\begin{array}{c}
-1 / 6 \\
k
\end{array}\right)\left(\begin{array}{c}
-5 / 6 \\
k
\end{array}\right)=\frac{\left(\begin{array}{c}
6 k \\
k k
\end{array}\right)\left(\begin{array}{c}
3 k \\
4
\end{array}\right)}{432^{k}}
\end{gathered}
$$

for all $k \in \mathbb{N}=\{0,1,2, \ldots\}$. In 2011 Z. W. Sun [Su11] showed further that

$$
\sum_{k=1}^{p-1} \frac{\left(\begin{array}{c}
2 k \\
k
\end{array}\right)^{2}}{16^{k}} \equiv\left(\frac{-1}{p}\right)-p^{2} E_{p-3}\left(\bmod p^{3}\right)
$$

and

$$
\sum_{k=1}^{(p-1) / 2} \frac{\left(\begin{array}{c}
2 k \\
k
\end{array}\right)^{2}}{16^{k}} \equiv\left(\frac{-1}{p}\right)+p^{2} E_{p-3}\left(\bmod p^{3}\right)
$$

where $E_{0}, E_{1}, E_{2}, \ldots$ are the Euler numbers given by

$$
E_{0}=1 \text {, and } E_{n}=-\sum_{k=1}^{\lfloor n / 2\rfloor}\left(\begin{array}{c}
n \\
2 k
\end{array}\right) E_{n-2 k}(n=1,2,3, \ldots) .
$$


He also conjectured that

$$
\begin{aligned}
& \sum_{k=0}^{p-1} \frac{\left(\begin{array}{c}
2 k \\
k
\end{array}\right)\left(\begin{array}{c}
3 k \\
k
\end{array}\right)}{(2 k+1) 27^{k}} \equiv\left(\frac{p}{3}\right)\left(\bmod p^{2}\right), \\
& \sum_{k=0}^{p-1} \frac{\left(\begin{array}{c}
4 k \\
2 k
\end{array}\right)\left(\begin{array}{c}
2 k \\
k
\end{array}\right)}{(2 k+1) 64^{k}} \equiv\left(\frac{-1}{p}\right)-3 p^{2} E_{p-3}\left(\bmod p^{3}\right), \\
& \sum_{k=0}^{p-1} \frac{\left(\begin{array}{c}
6 k \\
3 k
\end{array}\right)\left(\begin{array}{c}
3 k \\
k
\end{array}\right)}{(2 k+1) 432^{k}} \equiv\left(\frac{p}{3}\right)\left(\bmod p^{2}\right),
\end{aligned}
$$

which were confirmed by Z.-H. Sun [S16]. Note that Z.-W. Sun [Su14] determined

$$
\sum_{k=0}^{(p-3) / 2} \frac{\left(\begin{array}{c}
2 k \\
k
\end{array}\right)^{2}}{(2 k+1) 16^{k}} \text { and } \sum_{k=(p+1) / 2}^{p-1} \frac{\left(\begin{array}{c}
2 k \\
k
\end{array}\right)^{2}}{(2 k+1) 16^{k}}
$$

modulo $p^{3}$.

In this paper we first establish the following result.

Theorem 1.1. Let $p$ be any odd prime.

(i) We have

$$
\sum_{k=0}^{\lfloor 3 p / 4\rfloor} \frac{\left(\begin{array}{c}
2 k \\
k
\end{array}\right)^{2}}{16^{k}} \equiv \begin{cases}1\left(\bmod p^{3}\right) & \text { if } p \equiv 1(\bmod 4) \\
-1+p^{2} /\left(2\left(\begin{array}{c}
(p-3) / 2 \\
(p-3) / 4
\end{array}\right)^{2}\right)\left(\bmod p^{3}\right) & \text { if } p \equiv 3(\bmod 4) .\end{cases}
$$

(ii) For each $a=2,3,4, \ldots$, we have

$$
\sum_{k=0}^{\left\lfloor\frac{3}{4} p^{a}\right\rfloor} \frac{\left(\begin{array}{c}
2 k \\
k
\end{array}\right)^{2}}{16^{k}} \equiv\left(\frac{-1}{p^{a}}\right)\left(\bmod p^{3}\right) .
$$

Remark 1.1. . Part (i) in the case $p \equiv 1(\bmod 4)$ and part (ii) were conjectured by Sun [Su11].

Our second theorem is as follows.

Theorem 1.2. Let $p>3$ be a prime. Then we have

$$
\begin{gathered}
\sum_{k=0}^{(p-1) / 2} \frac{\left(\begin{array}{c}
2 k \\
k
\end{array}\right)\left(\begin{array}{c}
3 k \\
k
\end{array}\right)}{27^{k}} \equiv\left(\frac{p}{3}\right) \frac{2^{p}+1}{3}\left(\bmod p^{2}\right), \\
\sum_{k=0}^{(p-1) / 2} \frac{\left(\begin{array}{c}
6 k \\
3 k
\end{array}\right)\left(\begin{array}{c}
3 k \\
k
\end{array}\right)}{(2 k+1) 432^{k}} \equiv\left(\frac{p}{3}\right) \frac{3^{p}+1}{4}\left(\bmod p^{2}\right),
\end{gathered}
$$




$$
\sum_{k=0}^{(p-1) / 2} \frac{\left(\begin{array}{c}
4 k \\
2 k
\end{array}\right)\left(\begin{array}{c}
2 k \\
k
\end{array}\right)}{(2 k+1) 64^{k}} \equiv\left(\frac{-1}{p}\right) 2^{p-1}\left(\bmod p^{2}\right) .
$$

Remark 1.2. We are also able to show the congruence

$$
\sum_{k=0}^{(p-1) / 2} \frac{\left(\begin{array}{c}
2 k \\
k
\end{array}\right)\left(\begin{array}{c}
3 k \\
k
\end{array}\right)}{(2 k+1) 27^{k}} \equiv\left(\frac{p}{3}\right)\left(3^{p}+2-2^{p+1}\right)\left(\bmod p^{2}\right)
$$

for any prime $p>3$.

\section{Proof of Theorem 1.1}

Lemma 2.1. (Sun $[\operatorname{Su} 11,(1.4)]$ ) For any prime $p>3$ we have

$$
\sum_{k=1}^{(p-1) / 2} \frac{4^{k}}{k^{2}\left(\begin{array}{c}
2 k \\
k
\end{array}\right)} \equiv(-1)^{(p-1) / 2} 4 E_{p-3}(\bmod p) \text {. }
$$

Proof of Theorem 1.1(i). In view of (1.1), (1.2) has the following equation form:

$$
\sum_{k=(p+1) / 2}^{\lfloor 3 p / 4\rfloor} \frac{\left(\begin{array}{c}
2 k \\
k
\end{array}\right)^{2}}{16^{k}} \equiv-p^{2} E_{p-3}+\frac{1-(-1)^{(p-1) / 2}}{2} \cdot \frac{p^{2}}{2\left(\begin{array}{c}
(p-3) / 2 \\
\lfloor p / 4\rfloor
\end{array}\right)}\left(\bmod p^{3}\right) .
$$

By [Su11, Lemma 2.1],

$k\left(\begin{array}{c}2 k \\ k\end{array}\right)\left(\begin{array}{c}2(p-k) \\ p-k\end{array}\right) \equiv(-1)^{\lfloor 2 k / p\rfloor-1} 2 p\left(\bmod p^{2}\right)$ for all $k=1, \ldots, p-1$.

Thus

$$
\begin{aligned}
\sum_{k=(p+1) / 2}^{\lfloor 3 p / 4\rfloor} \frac{\left(\begin{array}{c}
2 k \\
k
\end{array}\right)^{2}}{16^{k}} & \equiv \sum_{k=(p+1) / 2}^{\lfloor 3 p / 4\rfloor} \frac{4 p^{2}}{k^{2}\left(\begin{array}{c}
2(p-k) \\
p-k
\end{array}\right)^{2} 16^{k}}=\sum_{j=\lfloor p / 4\rfloor+1}^{(p-1) / 2} \frac{4 p^{2}}{(p-j)^{2}\left(\begin{array}{c}
2 j \\
j
\end{array}\right)^{2} 16^{p-j}} \\
& \equiv \frac{p^{2}}{4} \sum_{j=\lfloor p / 4\rfloor+1}^{(p-1) / 2} \frac{16^{j}}{j^{2}\left(\begin{array}{c}
2 j \\
j
\end{array}\right)^{2}}\left(\bmod p^{3}\right)
\end{aligned}
$$

and hence we have reduced (2.2) to the following simpler form

$$
\sum_{k=\lfloor n / 2\rfloor+1}^{n} \frac{16^{k}}{k^{2}\left(\begin{array}{c}
2 k \\
k
\end{array}\right)^{2}} \equiv-4 E_{p-3}+\frac{1-(-1)^{n}}{\left(\begin{array}{c}
n-1 \\
\lfloor n / 2\rfloor
\end{array}\right)^{2}}(\bmod p)
$$

where $n=(p-1) / 2$.

For each $k=0, \ldots, n$, clearly

$$
\left(\begin{array}{l}
n \\
k
\end{array}\right) \equiv\left(\begin{array}{c}
-1 / 2 \\
k
\end{array}\right)=\frac{\left(\begin{array}{c}
2 k \\
k
\end{array}\right)}{(-4)^{k}}(\bmod p)
$$


Thus

$$
\sum_{k=\lfloor n / 2\rfloor+1}^{n} \frac{16^{k}}{k^{2}\left(\begin{array}{c}
2 k \\
k
\end{array}\right)^{2}} \equiv \sum_{k=\lfloor n / 2\rfloor+1}^{n} \frac{1}{k^{2}\left(\begin{array}{l}
n \\
k
\end{array}\right)^{2}} \equiv 4 \sum_{k=\lfloor n / 2\rfloor+1}^{n} \frac{1}{\left(\begin{array}{l}
n-1 \\
k-1
\end{array}\right)^{2}}(\bmod p) .
$$

Note that

$$
\sum_{k=\lfloor n / 2\rfloor+1}^{n} \frac{1}{\left(\begin{array}{c}
n-1 \\
k-1
\end{array}\right)^{2}}=\sum_{k=\lfloor n / 2\rfloor}^{n-1} \frac{1}{\left(\begin{array}{c}
n-1 \\
k
\end{array}\right)^{2}}=\frac{1}{2} \sum_{k=0}^{n-1} \frac{1}{\left(\begin{array}{c}
n-1 \\
k
\end{array}\right)^{2}}+\frac{1-(-1)^{n}}{4\left(\begin{array}{c}
n-1 \\
\lfloor n / 2\rfloor
\end{array}\right)^{2}}
$$

and

$$
\sum_{k=0}^{n-1} \frac{1}{\left(\begin{array}{c}
n-1 \\
k
\end{array}\right)^{2}}=\frac{2 n^{2}}{n+1} \sum_{k=1}^{n} \frac{1}{k\left(\begin{array}{c}
2 n+1-k \\
n-k
\end{array}\right)}
$$

(cf. [SWZ]). So we have

$$
\begin{aligned}
& \sum_{k=\lfloor n / 2\rfloor+1}^{n} \frac{16^{k}}{k^{2}\left(\begin{array}{c}
2 k \\
k
\end{array}\right)^{2}}-\frac{1-(-1)^{n}}{\left(\begin{array}{c}
n-1 \\
\lfloor n / 2\rfloor
\end{array}\right)^{2}} \\
\equiv & \frac{4 n^{2}}{n+1} \sum_{k=1}^{n} \frac{1}{k\left(\begin{array}{c}
2 n+1-k \\
n-k
\end{array}\right)} \equiv 2 \sum_{k=1}^{n} \frac{1}{k\left(\begin{array}{c}
-k \\
n-k
\end{array}\right)}(\bmod p) .
\end{aligned}
$$

Observe that

$$
\begin{aligned}
\sum_{k=1}^{n} \frac{1}{k\left(\begin{array}{c}
-k \\
n-k
\end{array}\right)} & =\sum_{k=1}^{n} \frac{(-1)^{n-k}}{k\left(\begin{array}{l}
n-1 \\
k-1
\end{array}\right)}=n \sum_{k=1}^{n} \frac{(-1)^{n-k}}{k^{2}\left(\begin{array}{l}
n \\
k
\end{array}\right)} \\
& \equiv \frac{(-1)^{n-1}}{2} \sum_{k=1}^{n} \frac{4^{k}}{k^{2}\left(\begin{array}{c}
2 k \\
k
\end{array}\right)}(\bmod p) .
\end{aligned}
$$

Therefore, with the help of Lemma 2.1, we finally obtain

$\sum_{k=\lfloor n / 2\rfloor+1}^{n} \frac{16^{k}}{k^{2}\left(\begin{array}{c}2 k \\ k\end{array}\right)^{2}}-\frac{1-(-1)^{n}}{\left(\begin{array}{c}n-1 \\ \lfloor n / 2\rfloor\end{array}\right)^{2}} \equiv(-1)^{n-1} \sum_{k=1}^{n} \frac{4^{k}}{k^{2}\left(\begin{array}{c}2 k \\ k\end{array}\right)} \equiv-4 E_{p-3}(\bmod p)$.

This proves (2.3) and hence (1.2) follows.

Now we give a lemma which is a natural extension of (1.1).

Lemma 2.2. Let $p>3$ be a prime and let a be any positive integer. Then

$$
\sum_{k=0}^{\left(p^{a}-1\right) / 2} \frac{\left(\begin{array}{c}
2 k \\
k
\end{array}\right)^{2}}{16^{k}} \equiv\left(\frac{-1}{p^{a}}\right)+\left(\frac{-1}{p^{a-1}}\right) p^{2} E_{p-3}\left(\bmod p^{3}\right)
$$


Proof. Theorem 1.2 of Sun $[\mathrm{Su} 13]$ states that for any $d=0, \ldots,(p-1) / 2$ we have

$$
\sum_{k=0}^{(p-1) / 2} \frac{\left(\begin{array}{c}
2 k \\
k
\end{array}\right)\left(\begin{array}{c}
2 k \\
k+d
\end{array}\right)}{16^{k}} \equiv\left(\frac{-1}{p}\right)+\frac{(-1)^{d}}{4} p^{2} E_{p-3}\left(d+\frac{1}{2}\right)\left(\bmod p^{3}\right),
$$

where $E_{p-3}(x)$ denotes the Euler polynomial of the degree $p-3$.

In the case $d=0$ this yields (1.1). Modifying this proof of (1.1) slightly we immediately get (2.5).

In 1852 , Kummer proved that for any $m, n \in \mathbb{N}$ the $p$-adic valuation of the binomial coefficient $\left(\begin{array}{c}m+n \\ m\end{array}\right)$ is equal to the number of carry-overs when performing the addition of $m$ and $n$ written in base $p$.

Lemma 2.3. Let $p$ be an odd prime and let $a \in \mathbb{Z}^{+}$. For any $k=$ $1,2, \ldots,\left(p^{a}-1\right) / 2$, we have

$$
\operatorname{ord}_{p}\left(\begin{array}{c}
p^{a}-k \\
\frac{p^{a}-1}{2}-k
\end{array}\right) \leq a-1
$$

Proof. It is well known that

$$
\operatorname{ord}_{p}(n !)=\sum_{j=1}^{\infty}\left\lfloor\frac{n}{p^{j}}\right\rfloor
$$

Thus

$$
\operatorname{ord}_{p}\left(\begin{array}{c}
p^{a}-k \\
\frac{p^{a}-1}{2}-k
\end{array}\right)=\sum_{j=1}^{a-1}\left(\left\lfloor\frac{p^{a}-k}{p^{j}}\right\rfloor-\left\lfloor\frac{\left(p^{a}+1\right) / 2}{p^{j}}\right\rfloor-\left\lfloor\frac{\left(p^{a}-1\right) / 2-k}{p^{j}}\right\rfloor\right)
$$

does not exceed $a-1$ as each term in the sum is at most one. This concludes the proof.

Proof of Theorem 1.1(ii). In view of Lemma 2.2, we just need to verify that

$$
\sum_{k=\left(p^{a}+1\right) / 2}^{\left\lfloor 3 p^{a} / 4\right\rfloor} \frac{\left(\begin{array}{c}
2 k \\
k
\end{array}\right)^{2}}{16^{k}} \equiv\left(\frac{-1}{p^{a-1}}\right) p^{2} E_{p-3}\left(\bmod p^{3}\right) .
$$

Let $k$ and $l$ be positive integers with $k+l=p^{a}$ and $0<l<p^{a} / 2$. Then

$$
\frac{\left(\begin{array}{c}
2 k \\
k
\end{array}\right)^{2}}{\left(\begin{array}{c}
2 p^{a}-2 \\
p^{a}-1
\end{array}\right)^{2}}=\frac{\left(2 p^{a}-2 l\right) !^{2}}{\left(2 p^{a}-2\right) !^{2}}\left(\frac{\left(p^{a}-1\right) !}{\left(p^{a}-l\right) !}\right)^{4}=\frac{\prod_{0<i<l}\left(p^{a}-i\right)^{4}}{\prod_{1<j<2 l}\left(2 p^{a}-j\right)^{2}}
$$

and hence

$$
\frac{\left(\begin{array}{c}
2 k \\
k
\end{array}\right)^{2}}{\left(\begin{array}{c}
2 p^{a}-2 \\
p^{a}-1
\end{array}\right)^{2}} \cdot \frac{(2 l-1) !^{2}}{(l-1) !^{4}}=\frac{\prod_{0<i<l}\left(1-p^{a} / i\right)^{4}}{\prod_{1<j<2 l}\left(1-2 p^{a} / j\right)^{2}} \equiv 1(\bmod p) .
$$


Note that

$$
\left(\begin{array}{c}
2 p^{a}-2 \\
p^{a}-1
\end{array}\right)^{2}=p^{2 a} \prod_{j=2}^{p^{a}-1}\left(\frac{2 p^{a}-j}{j}\right)^{2} \equiv p^{2 a}\left(\bmod p^{2 a+1}\right)
$$

and

$$
\left(\begin{array}{c}
2 k \\
k
\end{array}\right)^{2}=\left(\begin{array}{c}
p^{a}+\left(2 k-p^{a}\right) \\
0 p^{a}+k
\end{array}\right)^{2} \equiv\left(\begin{array}{c}
2 k-p^{a} \\
k
\end{array}\right)^{2}=0\left(\bmod p^{2}\right)
$$

by Lucas' theorem. So we have

$$
\frac{l^{2}}{4}\left(\begin{array}{c}
2 l \\
l
\end{array}\right)^{2}=\frac{(2 l-1) !^{2}}{(l-1) !^{4}} \not \equiv 0\left(\bmod p^{2 a}\right)
$$

and

$$
\left(\begin{array}{c}
2 k \\
k
\end{array}\right)^{2} \equiv p^{2 a} \frac{(l-1) !^{4}}{(2 l-1) !^{2}}=\frac{4 p^{2 a}}{l^{2}\left(\begin{array}{c}
2 l \\
l
\end{array}\right)^{2}}\left(\bmod p^{3}\right)
$$

Therefore

$$
\begin{aligned}
\sum_{k=\left(p^{a}+1\right) / 2}^{\left\lfloor 3 p^{a} / 4\right\rfloor} \frac{\left(\begin{array}{c}
2 k \\
k
\end{array}\right)^{2}}{16^{k}} & \equiv \sum_{k=\left(p^{a}+1\right) / 2}^{\left\lfloor 3 p^{a} / 4\right\rfloor} \frac{4 p^{2 a}}{16^{k}\left(p^{a}-k\right)^{2}\left(\begin{array}{c}
2\left(p^{a}-k\right) \\
p^{a}-k
\end{array}\right)^{2}} \\
& \equiv \frac{p^{2 a}}{4} \sum_{l=\left\lfloor p^{a} / 4\right\rfloor+1}^{\left(p^{a}-1\right) / 2} \frac{16^{l}}{l^{2}\left(\begin{array}{c}
2 l \\
l
\end{array}\right)^{2}}\left(\bmod p^{3}\right) .
\end{aligned}
$$

For $k=1, \ldots,\left(p^{a}-1\right) / 2$, clearly

$$
\begin{aligned}
\frac{\left(\begin{array}{c}
\left(p^{a}-1\right) / 2 \\
k
\end{array}\right)}{\left(\begin{array}{c}
2 k \\
k
\end{array}\right) /(-4)^{k}} & =\frac{\left(\begin{array}{c}
\left(p^{a}-1\right) / 2 \\
k
\end{array}\right)}{\left(\begin{array}{c}
-1 / 2 \\
k
\end{array}\right)}=\prod_{j=0}^{k-1} \frac{\left(p^{a}-1\right) / 2-j}{-1 / 2-j} \\
& =\prod_{j=0}^{k-1}\left(1-\frac{p^{a}}{2 j+1}\right) \equiv 1(\bmod p) .
\end{aligned}
$$

Thus

$$
\begin{aligned}
\sum_{k=\left(p^{a}+1\right) / 2}^{\left\lfloor 3 p^{a} / 4\right\rfloor} \frac{\left(\begin{array}{c}
2 k \\
k
\end{array}\right)^{2}}{16^{k}} & \equiv \frac{p^{2 a}}{4} \sum_{k=\left\lfloor p^{a} / 4\right\rfloor+1}^{\left(p^{a}-1\right) / 2} \frac{1}{k^{2}\left(\begin{array}{c}
\left(p^{a}-1\right) / 2 \\
k
\end{array}\right)^{2}} \\
& \equiv p^{2 a} \sum_{k=\left\lfloor p^{a} / 4\right\rfloor+1}^{\left(p^{a}-1\right) / 2} \frac{1}{\left(\begin{array}{c}
\left(p^{a}-3\right) / 2 \\
k-1
\end{array}\right)}\left(\bmod p^{3}\right) .
\end{aligned}
$$


So (2.6) is reduced to

$$
p^{2 a-2} \sum_{k=\left\lfloor p^{a} / 4\right\rfloor}^{\left(p^{a}-3\right) / 2} \frac{1}{\left(\begin{array}{c}
\left(p^{a}-3\right) / 2 \\
k
\end{array}\right)^{2}} \equiv-\left(\frac{-1}{p^{a-1}}\right) E_{p-3}(\bmod p) .
$$

If $p^{a} \equiv 1(\bmod 4)$, then $\left(p^{a}-3\right) / 2$ is odd and hence

$$
\sum_{k=\left\lfloor p^{a} / 4\right\rfloor}^{\left(p^{a}-3\right) / 2} \frac{1}{\left(\begin{array}{c}
\left(p^{a}-3\right) / 2 \\
k
\end{array}\right)^{2}}=\frac{1}{2} \sum_{k=0}^{\left(p^{a}-3\right) / 2} \frac{1}{\left(\begin{array}{c}
\left(p^{a}-3\right) / 2 \\
k
\end{array}\right)^{2}} .
$$

If $p^{a} \equiv 3(\bmod 4)$, then $a \in\{3,5, \ldots\}$ and

$$
\sum_{k=\left\lfloor p^{a} / 4\right\rfloor}^{\left(p^{a}-3\right) / 2} \frac{1}{\left(\begin{array}{c}
\left(p^{a}-3\right) / 2 \\
k
\end{array}\right)^{2}}=\frac{1}{2} \sum_{k=0}^{\left(p^{a}-3\right) / 2} \frac{1}{\left(\begin{array}{c}
\left(p^{a}-3\right) / 2 \\
k
\end{array}\right)^{2}}+\frac{1}{2} \cdot \frac{1}{\left(\begin{array}{c}
\left(p^{a}-3\right) / 2 \\
\left(p^{a}-3\right) / 4
\end{array}\right)^{2}} .
$$

In the case $p^{a} \equiv 3(\bmod 4)$, as the fractional parts of $\left(p^{a}-3\right) /(2 p)$ and $\left(p^{a}-3\right) /(4 p)$ are $(p-3) /(2 p)$ and $(p-3) /(4 p)$ respectively, we have

$$
\left\lfloor\frac{\left(p^{a}-3\right) / 2}{p}\right\rfloor=2\left\lfloor\frac{\left(p^{a}-3\right) / 4}{p}\right\rfloor
$$

and hence

$\operatorname{ord}_{p}\left(\begin{array}{l}\left(p^{a}-3\right) / 2 \\ \left(p^{a}-3\right) / 4\end{array}\right)^{2}=2 \sum_{j=1}^{a-1}\left(\left\lfloor\frac{\left(p^{a}-3\right) / 2}{p^{j}}\right\rfloor-2\left\lfloor\frac{\left(p^{a}-3\right) / 4}{p^{j}}\right\rfloor\right)<2 a-2$.

No matter $p^{a} \equiv 1(\bmod 4)$ or not, we always have

$$
p^{2 a-2} \sum_{k=\left\lfloor p^{a} / 4\right\rfloor}^{\left(p^{a}-3\right) / 2} \frac{1}{\left(\begin{array}{c}
\left(p^{a}-3\right) / 2 \\
k
\end{array}\right)^{2}} \equiv \frac{p^{2 a-2}}{2} \sum_{k=0}^{\left(p^{a}-3\right) / 2} \frac{1}{\left(\begin{array}{c}
\left(p^{a}-3\right) / 2 \\
k
\end{array}\right)^{2}}(\bmod p) .
$$

So (2.7) has the following equivalent form:

$$
p^{2 a-2} \sum_{k=0}^{\left(p^{a}-3\right) / 2} \frac{1}{\left(\begin{array}{c}
\left.p^{a}-3\right) / 2 \\
k
\end{array}\right)^{2}} \equiv-2\left(\frac{-1}{p^{a-1}}\right) E_{p-3}(\bmod p) .
$$

The identity (2.4) with $n=\left(p^{a}-1\right) / 2$ yields that

$$
\sum_{k=0}^{\left(p^{a}-3\right) / 2} \frac{1}{\left(\begin{array}{c}
\left(p^{a}-3\right) / 2 \\
k
\end{array}\right)^{2}}=\frac{2\left(\left(p^{a}-1\right) / 2\right)^{2}}{\left(p^{a}+1\right) / 2} \sum_{k=1}^{\left(p^{a}-1\right) / 2} \frac{1}{k\left(\begin{array}{c}
p^{a}-k \\
\left(p^{a}-1\right) / 2-k
\end{array}\right)} .
$$

So (2.8) is reduced to

$$
p^{2 a-2} \sum_{k=1}^{\left(p^{a}-1\right) / 2} \frac{1}{k\left(\begin{array}{c}
p^{a}-k \\
\left(p^{a}+1\right) / 2
\end{array}\right)} \equiv-2\left(\frac{-1}{p^{a-1}}\right) E_{p-3}(\bmod p) .
$$


In view of Lemma 2.3 , if $1 \leqslant k \leqslant\left(p^{a}-1\right) / 2$ and $p^{a-1} \nmid k$, then

$$
\frac{p^{2 a-2}}{k\left(\begin{array}{c}
p^{a}-k \\
\left(p^{a}+1\right) / 2
\end{array}\right)} \equiv 0(\bmod p) \text {. }
$$

Thus

$$
\begin{aligned}
p^{2 a-2} \sum_{k=1}^{\left(p^{a}-1\right) / 2} \frac{1}{k\left(\begin{array}{c}
p^{a}-k \\
\left(p^{a}+1\right) / 2
\end{array}\right)} & \equiv p^{2 a-2} \sum_{j=1}^{(p-1) / 2} \frac{1}{p^{a-1} j\left(\begin{array}{c}
p^{a}-p^{a-1} j \\
\left(p^{a}+1\right) / 2
\end{array}\right)} \\
& =\frac{p^{a}+1}{2} \sum_{j=1}^{(p-1) / 2} \frac{1}{j(p-j)\left(\begin{array}{c}
p^{a}-p^{a-1} j-1 \\
\left(p^{a}-1\right) / 2
\end{array}\right)} \\
& \equiv-\frac{1}{2} \sum_{j=1}^{(p-1) / 2} \frac{1}{j^{2}\left(\begin{array}{c}
p^{a}-p^{a-1} j-1 \\
\left(p^{a}-1\right) / 2
\end{array}\right)}(\bmod p) .
\end{aligned}
$$

For each $j=1, \ldots,(p-1) / 2$, by Lucas' theorem we have

$$
\begin{aligned}
\left(\begin{array}{c}
p^{a-1}(p-j)-1 \\
\left(p^{a}-1\right) / 2
\end{array}\right) & =\left(\begin{array}{c}
p^{a-1}(p-1-j)+p^{a-1}-1 \\
p^{a-1}(p-1) / 2+\left(p^{a-1}-1\right) / 2
\end{array}\right) \\
& \equiv\left(\begin{array}{c}
p-1-j \\
(p-1) / 2
\end{array}\right)\left(\begin{array}{c}
p^{a-1}-1 \\
\left(p^{a}-1\right) / 2
\end{array}\right) \\
& \equiv(-1)^{\left(p^{a-1}-1\right) / 2}\left(\begin{array}{c}
p-j-1 \\
(p-1) / 2
\end{array}\right)(\bmod p),
\end{aligned}
$$

also

$$
\begin{aligned}
\left(\begin{array}{c}
p-j-1 \\
(p-1) / 2
\end{array}\right) & =\left(\begin{array}{c}
p-1-j \\
(p-1) / 2-j
\end{array}\right)=(-1)^{(p-1) / 2-j}\left(\begin{array}{c}
-p+(p-1) / 2 \\
(p-1) / 2-j
\end{array}\right) \\
& \equiv(-1)^{(p-1) / 2-j}\left(\begin{array}{c}
(p-1) / 2 \\
j
\end{array}\right) \equiv(-1)^{(p-1) / 2-j}\left(\begin{array}{c}
-1 / 2 \\
j
\end{array}\right) \\
& =(-1)^{(p-1) / 2} \frac{\left(\begin{array}{c}
2 j \\
j
\end{array}\right)}{4^{j}}(\bmod p) .
\end{aligned}
$$

Therefore

$$
\begin{aligned}
& p^{2 a-2} \sum_{k=1}^{\left(p^{a}-1\right) / 2} \frac{1}{k\left(\begin{array}{c}
p^{a}-k \\
\left(p^{a}+1\right) / 2
\end{array}\right)} \equiv \frac{(-1)^{\left(p^{a-1}+1\right) / 2}}{2} \sum_{j=1}^{(p-1) / 2} \frac{1}{j^{2}\left(\begin{array}{c}
p-j-1 \\
(p-1) / 2
\end{array}\right)} \\
\equiv & \frac{(-1)^{\left(p^{a-1}+1\right) / 2}}{2}(-1)^{(p-1) / 2} \sum_{j=1}^{(p-1) / 2} \frac{4^{j}}{j^{2}\left(\begin{array}{c}
2 j \\
j
\end{array}\right)}(\bmod p) .
\end{aligned}
$$

This, together with (2.1), yields the desired (2.9).

The proof of Theorem 1.1(ii) is now complete. 


\section{Proof of Theorem 1.2}

Lemma 3.1. Let $p>3$ be a prime, and $m \in\{1,2, \ldots,(p-1) / 2\}$. For any $p$-adic integer $t$, we have

$$
\left(\begin{array}{c}
m+p t-1 \\
(p-1) / 2
\end{array}\right)\left(\begin{array}{c}
-1-p t-m \\
(p-1) / 2
\end{array}\right) \equiv \frac{p t}{m}\left(\bmod p^{2}\right) .
$$

Proof. Since

$$
\begin{aligned}
\left(\begin{array}{c}
m+p t-1 \\
(p-1) / 2
\end{array}\right) & =\frac{\prod_{r=0}^{m-1}(p t+r) \times \prod_{s=1}^{(p-1) / 2-m}(p t-s)}{((p-1) / 2) !} \\
& \equiv \frac{(m-1) ! p t(-1)^{(p-1) / 2-m}((p-1) / 2-m) !}{((p-1) / 2) !}\left(\bmod p^{2}\right),
\end{aligned}
$$

and

$$
\begin{aligned}
& \left(\begin{array}{c}
-m-p t-1 \\
(p-1) / 2
\end{array}\right)=\frac{\prod_{j=1}^{(p-1) / 2}(-m-p t-j)}{((p-1) / 2) !} \\
\equiv & \frac{(-1)^{(p-1) / 2}(m+1)(m+2) \cdots(m+(p-1) / 2)}{((p-1) / 2) !}(\bmod p),
\end{aligned}
$$

we have

$$
\begin{aligned}
& \left(\begin{array}{c}
m+p t-1 \\
(p-1) / 2
\end{array}\right)\left(\begin{array}{c}
-m-p t-1 \\
(p-1) / 2
\end{array}\right) \\
\equiv & \frac{p t(m-1) !(-1)^{m}((p-1) / 2-m) !(m+1)(m+2) \cdots(m+(p-1) / 2)}{((p-1) / 2) !^{2}} \\
= & \frac{p t}{m} \frac{(-1)^{m}((p-1) / 2-m) !(m+(p-1) / 2) !}{((p-1) / 2) !^{2}}=\frac{p t}{m}(-1)^{m} \frac{\left(\begin{array}{c}
p-1 \\
(p-1) / 2
\end{array}\right)}{\left(\begin{array}{c}
p-1 \\
(p-1) / 2+m
\end{array}\right)} \\
\equiv & \frac{p t}{m}(-1)^{m}(-1)^{(p-1) / 2}(-1)^{(p-1) / 2+m}=\frac{p t}{m}\left(\bmod p^{2}\right) .
\end{aligned}
$$

This concludes the proof.

Remark 3.1. Let $p>3$ be a prime and $m \in\{(p+1) / 2, \ldots, p-1\}$. For any $p$-adic integer $t$, by Lemma 3.1 we have

$$
\begin{aligned}
& \left(\begin{array}{c}
m+p t-1 \\
(p-1) / 2
\end{array}\right)\left(\begin{array}{c}
-1-p t-m \\
(p-1) / 2
\end{array}\right) \\
= & \left(\begin{array}{c}
(m-p)+p(t+1)-1 \\
(p-1) / 2
\end{array}\right)\left(\begin{array}{c}
-1-p(t+1)-(m-p) \\
(p-1) / 2
\end{array}\right) \\
\equiv & \frac{p(t+1)}{m-p} \equiv \frac{p(t+1)}{m}\left(\bmod p^{2}\right) .
\end{aligned}
$$


Lemma 3.2. Let $p>3$ be a prime. For $k \in\{1,2, \ldots, p-1\}$ and p-adic integer $t$, we have

$$
\left(\begin{array}{c}
p t \\
k
\end{array}\right)\left(\begin{array}{c}
-1-p t \\
k
\end{array}\right) \equiv-\frac{p^{2} t^{2}}{k^{2}}-\frac{p t}{k}\left(\bmod p^{3}\right) .
$$

Proof. This is almost trivial. In fact,

$$
\begin{aligned}
\left(\begin{array}{c}
p t \\
k
\end{array}\right)\left(\begin{array}{c}
-1-p t \\
k
\end{array}\right) & =\frac{p t}{p t-k}\left(\begin{array}{c}
-1+p t \\
k
\end{array}\right)\left(\begin{array}{c}
-1-p t \\
k
\end{array}\right) \\
& \equiv \frac{p t}{p t-k}\left(\begin{array}{c}
-1 \\
k
\end{array}\right)^{2}=\frac{p t\left(p^{2} t^{2}+p t k+k^{2}\right)}{(p t)^{3}-k^{3}} \\
& \equiv-\frac{p^{2} t^{2}}{k^{2}}-\frac{p t}{k}\left(\bmod p^{3}\right)
\end{aligned}
$$

This proves (3.2).

Recall that those $H_{n}=\sum_{0<k \leqslant n} 1 / k$ with $n \in \mathbb{N}$ are called harmonic numbers. If a prime $p$ does not divide an integer $a$, then we let $q_{p}(a)$ denote the Fermat quotient $\left(a^{p-1}-1\right) / p$.

Lemma 3.3. (Lemma $[\mathrm{L}]$ ). For any prime $p>3$, we have

$$
\begin{gathered}
H_{\lfloor p / 2\rfloor} \equiv-2 q_{p}(2)(\bmod p), H_{\lfloor p / 4\rfloor} \equiv-3 q_{p}(2)(\bmod p), \\
H_{\lfloor p / 3\rfloor} \equiv-\frac{3}{2} q_{p}(3)(\bmod p) \text { and } H_{\lfloor p / 6\rfloor} \equiv-2 q_{p}(2)-\frac{3}{2} q_{p}(3)(\bmod p),
\end{gathered}
$$

where $q_{p}(2)=\left(2^{p-1}-1\right) / p$ and $q_{p}(3)=\left(3^{p-1}-1\right) / p$ stand for the Fermat quotients.

For $n \in \mathbb{N}$, define

$S_{n}(x)=\sum_{k=0}^{n}\left(\begin{array}{l}x \\ k\end{array}\right)\left(\begin{array}{c}-1-x \\ k\end{array}\right)$ and $T_{n}(x)=\sum_{k=0}^{n}\left(\begin{array}{l}x \\ k\end{array}\right)\left(\begin{array}{c}-1-x \\ k\end{array}\right) \frac{1+2 x}{1+2 k}$.

By [S16, (2.2)] with $a=x+1$ and $b=0$, we have

$$
S_{n}(x)+S_{n}(x+1)=2\left(\begin{array}{l}
x \\
n
\end{array}\right)\left(\begin{array}{c}
-2-x \\
n
\end{array}\right) .
$$

By $[S 16,(2.2)]$ with $b=2$, we get

$$
T_{n}(x)-T_{n}(x-1)=2\left(\begin{array}{c}
x-1 \\
n
\end{array}\right)\left(\begin{array}{c}
-x-1 \\
n
\end{array}\right) .
$$

Proof of Theorem 1.2. For any $p$-adic integer $a$, we let $\langle a\rangle_{p}$ denote the least nonnegative integer $r$ with $a \equiv r(\bmod p)$. For convenience, we also set $n=(p-1) / 2$. 
(i) For any $p$-adic integer $a \not \equiv 0(\bmod p)$, by using (3.3) we get

$$
\begin{aligned}
& S_{n}(a)-(-1)^{\langle a\rangle_{p}} S_{n}\left(a-\langle a\rangle_{p}\right) \\
= & \sum_{k=0}^{\langle a\rangle_{p}-1}(-1)^{k}\left(S_{n}(a-k)+S_{n}(a-k-1)\right) \\
= & \sum_{k=0}^{\langle a\rangle_{p}-1}(-1)^{k} 2\left(\begin{array}{c}
a-k-1 \\
n
\end{array}\right)\left(\begin{array}{c}
k-a-1 \\
n
\end{array}\right)
\end{aligned}
$$

and hence

$$
\begin{aligned}
& S_{n}(a)-(-1)^{\langle a\rangle_{p}} S_{n}(p t) \\
= & 2 \sum_{k=0}^{\langle a\rangle_{p}-1}(-1)^{k}\left(\begin{array}{c}
\langle a\rangle_{p}+p t-k-1 \\
n
\end{array}\right)\left(\begin{array}{c}
-1-p t-\left(\langle a\rangle_{p}-k\right) \\
n
\end{array}\right),
\end{aligned}
$$

where $t:=\left(a-\langle a\rangle_{p}\right) / p$. By Lemma 3.2,

$$
S_{n}(p t)=\sum_{k=0}^{n}\left(\begin{array}{c}
p t \\
k
\end{array}\right)\left(\begin{array}{c}
-1-p t \\
k
\end{array}\right) \equiv 1-\sum_{k=1}^{n} \frac{p t}{k}=1-p t H_{n}\left(\bmod p^{2}\right) .
$$

So, with helps of Lemma 3.1 and Remark 3.1, we have

$$
S_{n}(a)-(-1)^{\langle a\rangle_{p}}\left(1-p t H_{n}\right) \equiv 2 \sum_{k=0}^{\langle a\rangle_{p}-1}(-1)^{k} \frac{p\left(t+\delta_{k}\right)}{\langle a\rangle_{p}-k}\left(\bmod p^{2}\right),
$$

where $\delta_{k}$ takes 1 or 0 according as $\langle a\rangle_{p}-k>p / 2$ or not.

Observe that

$$
\sum_{k=0}^{(p-1) / 2} \frac{\left(\begin{array}{c}
2 k \\
k
\end{array}\right)\left(\begin{array}{c}
3 k \\
k
\end{array}\right)}{27^{k}}=\sum_{k=0}^{n}\left(\begin{array}{c}
-1 / 3 \\
k
\end{array}\right)\left(\begin{array}{c}
-2 / 3 \\
k
\end{array}\right)=S_{n}(a)
$$

with $a=-1 / 3$. Note that

$$
\langle a\rangle_{p}= \begin{cases}(p-1) / 3 & \text { if } p \equiv 1(\bmod 3) \\ (2 p-1) / 3 & \text { if } p \equiv 2(\bmod 3)\end{cases}
$$

Hence

$$
t:=\frac{a-\langle a\rangle_{p}}{p}= \begin{cases}-1 / 3 & \text { if } p \equiv 1(\bmod 3) \\ -2 / 3 & \text { if } p \equiv 2(\bmod 3)\end{cases}
$$

Case 1. $p \equiv 1(\bmod 3)$. 
In this case, $\langle a\rangle_{p}=(p-1) / 3, t=-1 / 3$, and $\delta_{k}=0$ for all $k=$ $0, \ldots,\langle a\rangle_{p}-1$. So we have

$$
\begin{aligned}
& S_{n}\left(-\frac{1}{3}\right)-(-1)^{(p-1) / 3}\left(1-p t H_{n}\right) \\
\equiv & 2 p t(-1)^{(p-1) / 3} \sum_{j=1}^{(p-1) / 3} \frac{(-1)^{j}}{j}=2 p t\left(H_{(p-1) / 6}-H_{(p-1) / 3}\right)\left(\bmod p^{2}\right) .
\end{aligned}
$$

Combining this with Lemma 3.3 and recalling that $t=-1 / 3$, we immediately obtain the desired congruence

$$
S_{n}\left(-\frac{1}{3}\right) \equiv 1+\frac{2}{3} p q_{p}(2)\left(\bmod p^{2}\right)
$$

Case 2. $p \equiv 2(\bmod 3)$.

In this case, we have $\langle a\rangle_{p}=(2 p-1) / 3, t=-2 / 3$ and

$$
\delta_{k}= \begin{cases}1 & \text { if } 0 \leq k<(p+1) / 6 \\ 0 & \text { if }(p+1) / 6 \leq k \leq\langle a\rangle_{p}-1\end{cases}
$$

So we have

$$
\begin{aligned}
& S_{n}\left(-\frac{1}{3}\right)-(-1)^{(2 p-1) / 3}\left(1-p t H_{n}\right) \\
\equiv & 2 p(t+1) \sum_{k=0}^{(p-5) / 6} \frac{(-1)^{k}}{\langle a\rangle_{p}-k}+2 p t \sum_{k=(p+1) / 6}^{(2 p-4) / 3} \frac{(-1)^{k}}{\langle a\rangle_{p}-k} \\
= & 2 p(t+1)(-1)^{(2 p-1) / 3} \sum_{j=(p+1) / 2}^{(2 p-1) / 3} \frac{(-1)^{j}}{j}+2 p t(-1)^{(2 p-1) / 3} \sum_{j=1}^{(p-1) / 2} \frac{(-1)^{j}}{j} \\
= & -2 p(t+1) \sum_{j=1}^{(2 p-1) / 3} \frac{(-1)^{j}}{j}+2 p \sum_{j=1}^{(p-1) / 2} \frac{(-1)^{j}}{j} \\
= & -2 p(t+1)\left(H_{\lfloor p / 3\rfloor}-H_{\lfloor 2 p / 3\rfloor}\right)+2 p\left(H_{\lfloor p / 4\rfloor}-H_{\lfloor p / 2\rfloor}\right)\left(\bmod p^{2}\right) .
\end{aligned}
$$

Note that

$$
H_{\lfloor 2 p / 3\rfloor}=\sum_{k=1}^{(p-1) / 2}\left(\frac{1}{k}+\frac{1}{p-k}\right)-\sum_{j=1}^{(p-1) / 3} \frac{1}{p-j} \equiv H_{\lfloor p / 3\rfloor}(\bmod p) .
$$

Therefore,

$$
S_{n}\left(-\frac{1}{3}\right)+1-p t H_{\lfloor p / 2\rfloor} \equiv 2 p\left(H_{\lfloor p / 4\rfloor}-H_{\lfloor p / 2\rfloor}\right)\left(\bmod p^{2}\right) .
$$


This, together with Lemma 3.3 and the fact that $t=-2 / 3$, yields the desired congruence

$$
S_{n}\left(-\frac{1}{3}\right) \equiv-1-\frac{2}{3} p q_{p}(2)\left(\bmod p^{2}\right)
$$

In view of the above, we have completed the proof of (1.4).

(ii) For any $p$-adic integer $a$ with $a(2 a+1) \not \equiv 0(\bmod p)$, if we set $t=\left(a-\langle a\rangle_{p}\right) / p$ then by (3.4) we have

$$
\begin{aligned}
T_{n}(a)-T_{n}(p t) & =\sum_{k=1}^{\langle a\rangle_{p}}\left(T_{n}(a-k+1)-T_{n}(a-k)\right) \\
& =\sum_{k=1}^{\langle a\rangle_{p}} 2\left(\begin{array}{c}
a-k \\
n
\end{array}\right)\left(\begin{array}{c}
k-a-2 \\
n
\end{array}\right) \\
& =2 \sum_{k=1}^{\langle a\rangle_{p}}\left(\begin{array}{c}
m_{k}+p t-1 \\
n
\end{array}\right)\left(\begin{array}{c}
-1-p t-m_{k} \\
n
\end{array}\right),
\end{aligned}
$$

where $m_{k}=\langle a\rangle_{p}-k+1$. In view of Lemmas 3.2 and 3.3,

$$
\begin{aligned}
T_{n}(p t)-(1+2 p t) & =\sum_{k=0}^{n}\left(\begin{array}{c}
p t \\
k
\end{array}\right)\left(\begin{array}{c}
-1-p t \\
k
\end{array}\right) \frac{1+2 p t}{1+2 k}-(1+2 p t) \\
& \equiv\left(\begin{array}{c}
p t \\
n
\end{array}\right)\left(\begin{array}{c}
-1-p t \\
n
\end{array}\right) \frac{1+2 p t}{p}-\sum_{k=1}^{n-1} \frac{p t}{k(1+2 k)} \\
& \equiv\left(-\frac{p^{2} t^{2}}{n^{2}}-\frac{p t}{n}\right) \frac{1+2 p t}{p}-\sum_{k=1}^{n-1} \frac{p t}{k(1+2 k)} \\
& \equiv 2 t+2 p t-p t \sum_{k=1}^{n-1} \frac{1}{k}+2 p t \sum_{k=1}^{n-1} \frac{1}{2 k+1} \\
& \equiv 2 t-2 p t-p t H_{n}+2 p t\left(H_{p-1}-\frac{H_{n}}{2}\right) \\
& \equiv 2 t-2 p t+4 p t q_{p}(2)\left(\bmod p^{2}\right)
\end{aligned}
$$

and hence

$$
T_{n}(p t) \equiv 1+2 t+4 p t q_{p}(2)\left(\bmod p^{2}\right) .
$$


Therefore, with the helps of Lemma 3.1 and Remark 3.1, we have

$$
\begin{aligned}
& T_{n}(a)-\left(1+2 t+4 p t q_{p}(2)\right) \\
\equiv & 2 \sum_{k=1}^{\langle a\rangle_{p}}\left(\begin{array}{c}
m_{k}+p t-1 \\
n
\end{array}\right)\left(\begin{array}{c}
-1-p t-m_{k} \\
n
\end{array}\right) \\
\equiv & 2 \sum_{k=1}^{\langle a\rangle_{p}} \frac{p\left(t+\delta_{k}\right)}{m_{k}}=2 \sum_{j=1}^{\langle a\rangle_{p}} \frac{p t}{j}+2 \sum_{\substack{j=1 \\
j>p / 2}}^{\langle a\rangle_{p}} \frac{1}{j}\left(\bmod p^{2}\right),
\end{aligned}
$$

where $\delta_{k}$ takes 1 or 0 according as $m_{k}>p / 2$ or not. Below we deal with $a=-1 / 6,-1 / 4$.

Clearly,

$$
H_{p-k}=H_{p-1}-\sum_{0<j<k} \frac{1}{p-j} \equiv H_{k-1}(\bmod p)
$$

for all $k=1, \ldots, p-1$. Thus, with the help of Lemma 3.3 we have

$$
H_{\lfloor 3 p / 4\rfloor} \equiv H_{p-1-\lfloor 3 p / 4\rfloor}=H_{\lfloor p / 4\rfloor} \equiv-3 q_{p}(2)(\bmod p)
$$

and

$$
H_{\lfloor 5 p / 6\rfloor} \equiv H_{p-1-\lfloor 5 p / 6\rfloor}=H_{\lfloor p / 6\rfloor} \equiv-2 q_{p}(2)-\frac{3}{2} q_{p}(3)(\bmod p)
$$

Case I. $\langle a\rangle_{p}<n$.

If $a=-1 / 6$, then $p \equiv 1(\bmod 6),\langle a\rangle_{p}=(p-1) / 6$ and $t=-1 / 6$. By the above,

$$
\begin{aligned}
T_{n}\left(-\frac{1}{6}\right) & \equiv \frac{2}{3}-\frac{2}{3} p q_{p}(2)-\frac{p}{3} H_{\lfloor p / 6\rfloor} \\
& \equiv \frac{2}{3}-\frac{2}{3} p q_{p}(2)-\frac{p}{3}\left(-2 q_{p}(2)-\frac{3}{2} q_{p}(3)\right) \\
& \equiv \frac{2}{3}+\frac{p}{2} q_{p}(3)\left(\bmod p^{2}\right)
\end{aligned}
$$

and thus

$$
\sum_{k=0}^{n} \frac{\left(\begin{array}{c}
6 k \\
3 k
\end{array}\right)\left(\begin{array}{c}
3 k \\
k
\end{array}\right)}{(2 k+1) 432^{k}}=\frac{3}{2} T_{n}\left(-\frac{1}{6}\right) \equiv 1+\frac{3}{4} p q_{p}(3)=\frac{3^{p}+1}{4}\left(\bmod p^{2}\right) .
$$


If $a=-1 / 4$, then $p \equiv 1(\bmod 4),\langle a\rangle_{p}=(p-1) / 4$ and $t=-1 / 4$. By the above,

$$
\begin{aligned}
T_{n}\left(-\frac{1}{4}\right) & \equiv \frac{1}{2}-p q_{p}(2)-\frac{p}{2} H_{\lfloor p / 4\rfloor} \\
& \equiv \frac{1}{2}-p q_{p}(2)-\frac{p}{2}\left(-3 q_{p}(2)\right) \\
& \equiv \frac{1}{2}+\frac{p}{2} q_{p}(2)\left(\bmod p^{2}\right)
\end{aligned}
$$

and thus

$$
\sum_{k=0}^{n} \frac{\left(\begin{array}{c}
4 k \\
2 k
\end{array}\right)\left(\begin{array}{c}
2 k \\
k
\end{array}\right)}{(2 k+1) 64^{k}}=2 T_{n}(-1 / 4) \equiv 1+p q_{p}(2)=2^{p-1}\left(\bmod p^{2}\right) .
$$

Case II. $\langle a\rangle_{p}>n$.

If $a=-1 / 6$, then $p \equiv 5(\bmod 6),\langle a\rangle_{p}=(5 p-1) / 6$ and $t=-5 / 6$. By the above,

$$
\begin{aligned}
T_{n}\left(-\frac{1}{6}\right) & \equiv-\frac{2}{3}+\frac{2}{3} p q_{p}(2)+\frac{p}{3} H_{\lfloor 5 p / 6\rfloor} \\
& \equiv-\frac{2}{3}+\frac{2}{3} p q_{p}(2)+\frac{p}{3}\left(-2 q_{p}(2)-\frac{3}{2} q_{p}(3)\right) \\
& \equiv-\frac{2}{3}-\frac{p}{2} q_{p}(3)\left(\bmod p^{2}\right)
\end{aligned}
$$

and hence

$$
\sum_{k=0}^{n} \frac{\left(\begin{array}{c}
6 k \\
3 k
\end{array}\right)\left(\begin{array}{c}
3 k \\
k
\end{array}\right)}{(2 k+1) 432^{k}}=\frac{3}{2} T_{n}\left(-\frac{1}{6}\right) \equiv-1-\frac{3}{4} p q_{p}(3)=-\frac{3^{p}+1}{4}\left(\bmod p^{2}\right) .
$$

If $a=-1 / 4$, then $p \equiv 3(\bmod 4),\langle a\rangle_{p}=(3 p-1) / 4$ and $t=-3 / 4$. So

$$
\begin{aligned}
T_{n}\left(-\frac{1}{4}\right) & \equiv-\frac{1}{2}+p q_{p}(2)+\frac{p}{2} H_{\lfloor 3 p / 4\rfloor} \\
& \equiv-\frac{1}{2}+p q_{p}(2)+\frac{p}{2}\left(-3 q_{p}(2)\right) \\
& \equiv-\frac{1}{2}-\frac{p}{2} q_{p}(2)\left(\bmod p^{2}\right)
\end{aligned}
$$

and hence

$$
\sum_{k=0}^{n} \frac{\left(\begin{array}{c}
4 k \\
2 k
\end{array}\right)\left(\begin{array}{c}
2 k \\
k
\end{array}\right)}{(2 k+1) 64^{k}}=2 T_{n}\left(-\frac{1}{4}\right) \equiv-1-p q_{p}(2)=-2^{p-1}\left(\bmod p^{2}\right),
$$

The proof of Theorem 1.2 is now complete. 
Acknowledgment. The first author would like to thank Dr. Hao Pan for help comments.

\section{REFERENCES}

[L] E. Lehmer, On congruences involving Bernoulli numbers and the quotients of Fermat and Wilson, Ann. Math. 39(1938), 350-360.

[M1] E. Mortenson, A supercongruence conjecture of Rodriguez-Villegas for a certain truncated hypergeometric function, J. Number Theory 99 (2003), 139-147.

[M2] E. Mortenson, Supercongruences for truncated ${ }_{n+1} F_{n}$ hypergeometric series with applications to certain weight three newforms, Proc. Amer. Math. Soc. 133 (2005), 321-330.

[RV] F. Rodriguez-Villegas, Hypergeometric families of Calabi-Yau manifolds. Calabi-Yau Varieties and Mirror Symmetry (Yui, Noriko (ed.) et al., Toronto, ON, 2001), 223-231, Fields Inst. Commun., 38, Amer. Math. Soc., Providence, RI, (2003).

[S16] Z.-H. Sun, Super congruences involving Bernoulli polynomials, Int. J. Number Theory, in press.

[Su11] Z.-W. Sun, Super congruences and Euler numbers, Sci. China Math. 54 (2011), 2509-2535.

[Su13] Z.-W. Sun, Supercongruences involving products of two binomial coefficients, Finite Fields Appl. 22 (2013), 24-44.

[Su14] Z.-W. Sun, p-adic congruences motivated by series, J. Number Theory 134 (2014), no.1, 181-196.

[SWZ] B. Sury, T.-M. Wang and F.-Z. Zhao, Identities involving reciprocals of binomial coefficients, J. Integer Seq. 7 (2004), Article 04.2.8.

(Guo-Shuai Mao) Department of Mathematics, Nanjing University, Nanjing 210093, People's Republic of China

E-mail address: mg1421007@smail.nju.edu.cn

(Zhi-Wei Sun) Department of Mathematics, Nanjing University, NanJing 210093, People's Republic of China

E-mail address: zwsun@nju.edu.cn 